\title{
Dresdner Dermatologische Demonstration 2004 - zugleich Tagung der Sächsischen Dermatologischen Gesellschaft in Verbindung mit der 1. Internationalen Dermatologen-Tagung Dresden - Prag - Wroclaw, 23. -24. April 2004
}

\section{E. Köstler \\ G. Hansel \\ U. Wollina}

\author{
Conference Report: Dresden Dermatology Demonstration 2004 - Meeting of \\ the Saxonian Society of Dermatology in Association with the 1st International \\ Dermatology Symposium Dresden - Prague - Wroclaw, April 23-24, 2004
}

Vorsitz: Prof. Dr. U. Wollina (Dresden), Prof. E. Baran (Wroclaw), Prof. J. Hercogova (Prag)

Berichterstatter: Prof. Dr. E. Köstler, Dipl.-Med. G. Hansel, Prof. Dr. U. Wollina

Histopathologie: Frau Dr. J. Schönlebe, PD Dr. med. G. Haroske Klinische Fotodokumentation: Frau R. Herz

\section{Einleitung}

Die Sächsische Dermatologische Gesellschaft hatte sich die Aufgabe gestellt, schon in Vorbereitung der EU-Osterweiterung die Kontakte zu den polnischen und tschechischen Dermatologen im Dreiländer-Dreieck wieder aufzunehmen und zu intensivieren. Diese Idee wurde von den Kollegen aus Wroclaw und Prag sehr positiv aufgenommen, so dass die 1 . Internationale Dermatologen-Tagung quasi am Vorabend des offiziellen Beitritts Polens und Tschechiens in die EU stattfinden konnte.

Die Tagung wurde durch ein Grußwort des Präsidenten der International Society of Dermatology Coleman Jacobson eröffnet, der einen kurzen historischen Abriss der ISD gab. Den dermatologischen Part eröffnete J. Hercogová (Prag). Sie stellte die typischen und zahlreichen atypischen klinischen Formen der Borreliose dar. Süddeutschland, Tschechien und die Slowakei sind bekanntermaßen Endemiegebiete. E. Baran (Wroclaw) stellte eigene Ergebnisse sowie die aktuelle Literatur zur Kombinationstherapie der Onychomykose vor. Abheilungsraten (klinisch wie mykologisch) sind höher, wenn zusätzlich zur systemischen Behandlung ciclopiroxolamin-haltiger Nagellack eingesetzt wird. J. Smetana (Prag) sprach zu glykobiologischen Aspekten der epidermalen Differenzierung und den Aberrationen im Rahmen der Hautkarzinogenese. Weitere Beiträge beschäftigen sich mit der gonorrhoischen Proktitis (A. Nechvatal, Prag), der Dermatochirurgie der Lidtumoren (A. Bienek, Wroclaw), der 20-MHz-Sonographie der Haut, der Epidemiologie blasenbildender Dermatosen und der Moulagensammlung in Wroclaw (Bialinicki-Birula, Wroclaw). E. Köstler (Dresden) gab eine Übersicht zu den kutanen Porphyrien, die ein Schwerpunkt der Dresden-Friedrichstädter Klinik darstellen. Er verwies insbesondere auf neue molekularbiologische und therapeutische Aspekte. M. Sticherling (Leipzig) betonte die Rolle des Dermatologen bei den so genannten Kollagenosen. Eine interessante Studie zum Pruritus bei Psoriasis wurde von J. Szepietowski (Wroclaw) vorgestellt. J. Glander (Leipzig) gab eine Übersicht zu den In-vitro-Verfahren der Fertilitätsprüfungen aus andrologischer Sicht. Diese Veranstaltung soll Auftakt zu regelmäßigen 2-jährigen Treffen sein. Das nächste ist für 2006 in Prag geplant.

\section{Gastvorträge}

J. Simon (Leipzig): Aktinische Präkanzerosen, Basalzellkarzinome - neue Erkenntnisse, moderne Therapien.

A. Hackert, M. Meuer (Dresden): Laser in der Dermatologie.

\section{Infantile digitale Fibromatose}

\section{G. Hansel, U. Gabsch, J. Schönlebe}

\section{Anamnese}

Im Mai vergangenen Jahres stellten die Eltern den damals Fünfjährigen mit einem derben, nicht schmerzhaften Tumor an der fünften Zehe des rechten Fußes vor. Dieser Tumor war innerhalb eines Jahres gewachsen. Innerhalb eines halben Jahres (Oktober 2003) vergrößerte sich der Knoten und in unmittelbarer Umgebung bildete sich ein zweiter, kleinerer, klinisch identischer Tumor aus (Abb.1). Es erfolgte eine diagnostische Exstirpation des kleineren Knotens. Bis Dezember 2003 hatte sich im 4. Zehenzwischenraum rechts ein weiteres Knötchen entwickelt. 


\section{Hautbefund}

Wir sahen an der Ober- und Außenseite der 5. Zehe rechts einen $12 \times 12 \mathrm{~mm}$ großen, rötlich-hautfarbenen, derben, indolenten halbkugeligen Tumor. Proximal davon, in Höhe des Grundgelenkes fand sich an der Außenseite der Zehe ein weiteres, $9 \times 8 \mathrm{~mm}$ großes Knötchen und im Zehenzwischenraum IV ein ca. $2 \mathrm{~mm}$ großes Knötchen gleichen Aussehens.

\section{Histologie}

Subepidermal im korialen Bindegewebe ein umschriebenes, knotiges Infiltrat aus in Zügen angeordneten Spindelzellen mit teils spindeligen, teils zigarrenförmigen Zellkernen mit aufgelockertem Chromatingerüst. Positive Reaktion für glatt-muskuläres Actin als Nachweis für Myofibroblasten.

\section{Therapie und Verlauf}

Bei der diagnostischen Exzision wurde der kleinere der beiden ursprünglichen Tumoren in toto entfernt. Für den größeren Knoten empfahlen wir bei bisher asymptomatischem Verlauf ein weiterhin abwartendes Verhalten. Der bei einer operativen Entfernung des Tumors entstehende Defekt könnte nur durch eine Hauttransplantation verschlossen werden. Bei dem zu erwartenden Längenwachstum müsste an der Phalanx sowohl mit funktionell als auch kosmetisch ungünstigem Operationsergebnis gerechnet werden, weshalb bei Beschwerdefreiheit Wait-and-See empfohlen wurde. An der Exzisionsstelle hatte sich ein Keloid ausgebildet.

\section{Kommentar}

Die infantile digitale Fibromatose ist selten. Klinisch ist sie durch derbe, indolente, lividrote Knoten charakterisiert. Diese treten gleich nach der Geburt bzw. im ersten Lebensjahr in Ein- oder Mehrzahl auf und sind in der Regel an den Streckseiten von Fingern und Zehen mit Bevorzugung der Endphalangen lokalisiert. Sie vergrößern sich langsam und können durch den Zug des straffen Bindegewebes zu Deformierungen, insbesondere zu Subluxationen der Endphalangen führen. Rezidive nach operativer Entfernung sind ebenso möglich wie spontane Rückbildungen. Lokalisation und histologisches Bild sprechen für eine reaktive Hyperplasie, zu der eine individuelle Disposition besteht und die bedingt durch eine periphere Mangeldurchblutung auf die Akren beschränkt zu sein scheint [1,2]. Die Entwicklung eines keloidartigen Plaques an der Exzisionsstelle spricht für diese

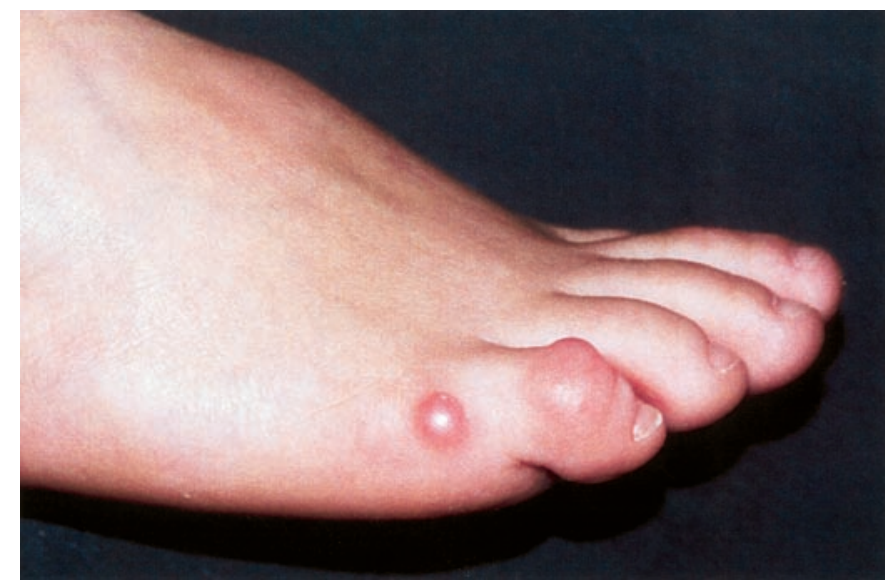

Abb. 1 Infantile digitale Fibromatose.
Annahme. Im Zytoplasma der Fibroblasten sind runde und ovale Körperchen nachweisbar, die mit der PAS-Reaktion nicht darstellbar sind, sich aber mit Eisen-Hämatoxylin tief purpurrot färben. Die Zellen exprimieren Vimentin und glatt-muskuläres Actin, welches sie als Myofibroblasten charakterisiert [3]. Differenzialdiagnostisch kommen u.a. ein kutanes Lymphozytom, Perniones, Dermatofibrome, benigne und maligne fibröse Histiozytome, Dermatofibrosarcoma protuberans und das Riesenzellfibroblastom in Betracht.

\section{Literatur}

1 Albrecht-Nebe H. Kasuistischer Beitrag zur infantilen digitalen Fibromatose. Dermatol Monatsschr 1983; 169:110-119

2 Falco NA, Upton J. Infantile digital fibromas. J Hand Surg (Am) 1995; 20: $1014-1020$

3 Mentzel T, Katenkamp D. Myofibroblastäre Tumoren: Kurzgefaßte Übersicht zur Klinik, Diagnose und Differentialdiagnose. Pathologe 1998; $19: 176-186$

\section{Granulomatöses kutanes T-Zell-Lymphom}

(T2 N1 M0 B0, Stadium IIa nach UICC 1993) -

Therapie mit pegyliert-liposomal verkapseltem Doxorubizin

\section{H. Konrad, W. Schmelz, U. Wollina}

\section{Anamnese}

Bei dem 83-jährigen Patienten seit 10/99 erste plaqueartige Hautveränderungen, schuppend, gelegentlich Pruritus. Therapie mit Creme-PUVA, darunter Besserung. Rezidiv 11/00, daraufhin Bade-PUVA Therapie und Röntgen-Weichteilbestrahlung des Tumors im Bereich der Wangenschleimhaut (10,0 Gy). Erneutes Rezidiv 08/01-MTX p. o., 25 mg. Rezidive 09/02 und 5/03 - jeweils Besserung unter PUVA-Therapie. 11/03 erneutes Rezidiv.

\section{Hautbefund}

Disseminiert am Hautorgan einschließlich Gesicht unterschiedlich große erythematöse Plaques, stark infiltiert, schuppend (ca. $30 \%$ KÖF) (Abb. 2). Onychodystrophie. Inguinale Lymphadenopathie.

\section{Labor}

Bis auf eine leichte Thrombozytose (361 Tpt/l) unauffällig.

\section{Histologie}

Wechselnd dichtes, in unterschiedlichem Maße epidermotrop sowie betont angiotropes Infiltrat der bekannten Mycosis fungoides. Den neoplastischen Zellen sind reichlich Entzündungszellen beigemischt. Insbesondere in den tiefen Koriumabschnitten zeigt das Infiltrat einen betont epitheloidzellig granulomatösen Charakter, auch mit Nachweis von einzelnen mehrkernigen Riesenzellen, im Sinne eines Gestaltwandels zu einer granulomatösen Form der Mycosis fungoides (Abb. 3a und b).

\section{Therapie und Verlauf}

Wir leiteten eine interne Monotherapie mit pegyliert-liposomal verkapseltem Doxorubizin (Caelyx ${ }^{\circledR}$ ) 20 mg/m² KÖF einmal monatlich ein. Nach 5 Zyklen partielle Remission. Keine Nebenwirkungen klinisch wie laborchemisch. 


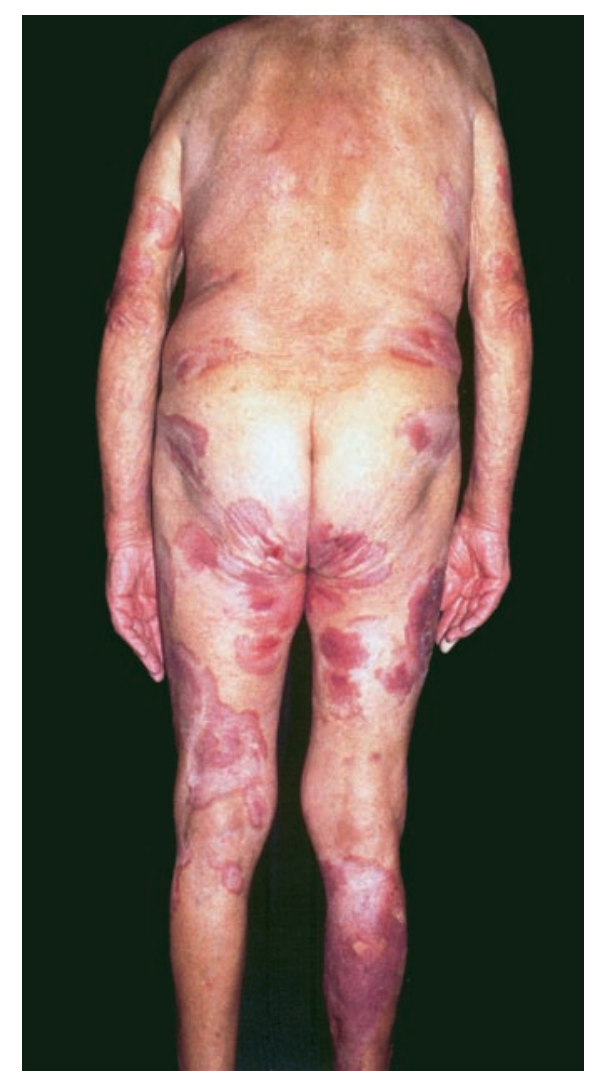

Abb. 2 Granulomatöse Mycosis fungoides.

\section{Kommentar}

Die granulomatösen Formen der kutanen T-Zell-Lymphome lassen sich i. W. in die Sonderform Granulomatous Slack Skin und granulomatöse Mycosis fungoides einordnen [3]. Erstere scheint auf Interferon-alpha und Strahlentherapie gut anzusprechen [6]. Die granulomatöse Mycosis fungoides nimmt einen teilweise sehr aggressiven Verlauf mit raschem Übergang in ein Tumorstadium unter Infiltration innerer Organe $[3,4]$.

Caelyx ${ }^{\circledR}$ ist eine pegylierte liposomale Formulierung von Doxorubicin-Hydrochlorid mit langer Zirkulationszeit, jedoch unter Vermeidung von Serum- oder Plasma-Peaks. Das Nebenwirkungsprofil ist deutlich besser als beim unverkapselten Wirkstoff, was insbesondere die kardiale Toxizität betrifft. Die Monotherapie mit Caelyx ${ }^{\circledR}$ ist den klassischen Polychemotherapie-Protokollen (KNOSPE/CHOPP) bezüglich Lebensqualität deutlich überlegen. In multizentrischen Studien wurden Ansprechraten von 88,2\% erzielt [5].

Bei kutanen Lymphom-Patienten mit Tumorprogress nach mehrfachen Vorbehandlungen ist liposomal verkapseltes Doxorubizin eine wirksame Option in der Second-Line-Therapie [1, 2]. Dies ist der erste Fall einer granulomatösen Mycosis fungoides, der mit Caelyx ${ }^{\circledR}$ behandelt wurde.
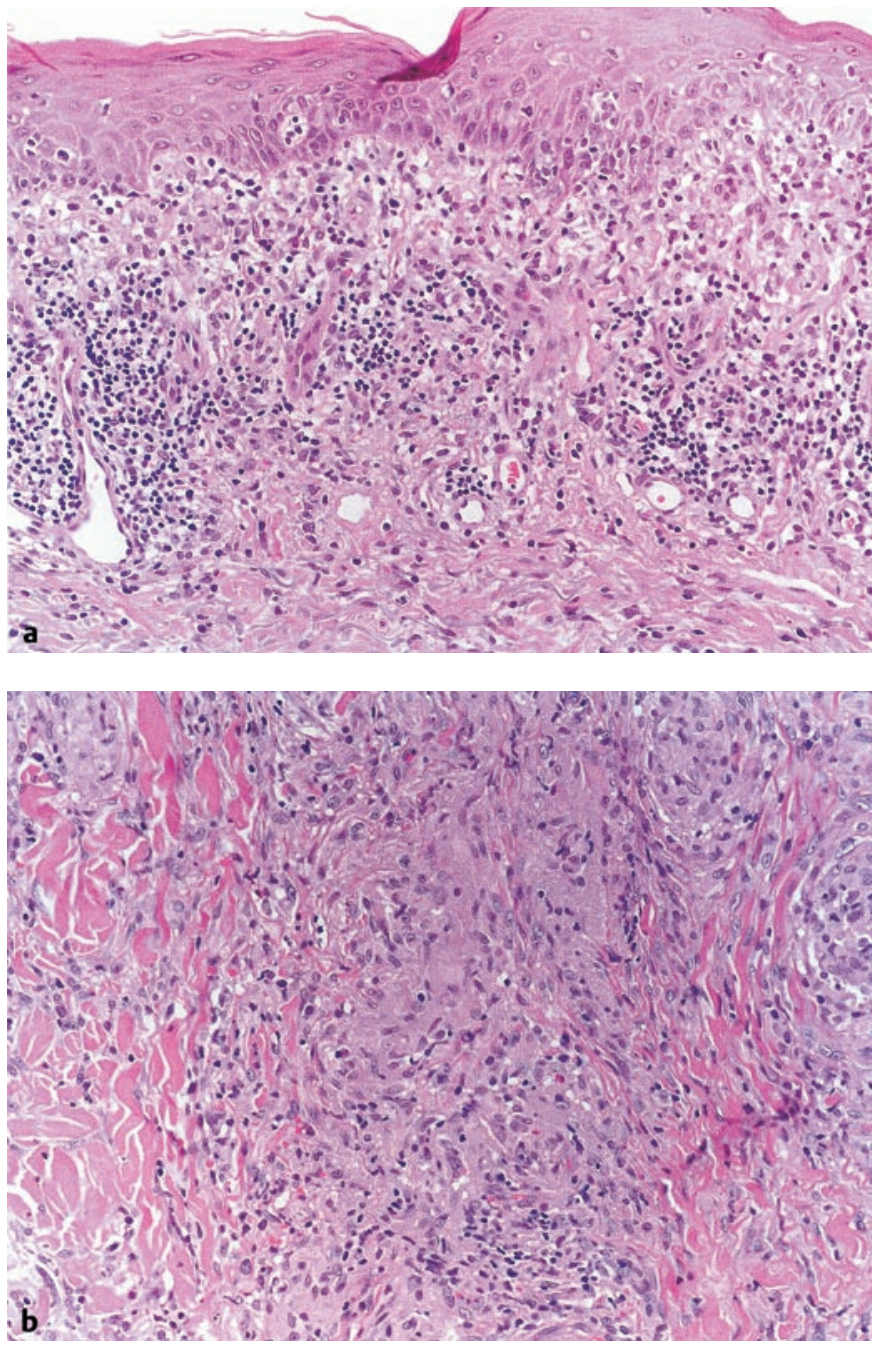

Abb 3 a Mycosis fungoides mit granulomatöser Umwandlung, Übersicht (HE). b Mycosis fungoides mit granulomatöser Umwandlung, Detail (HE).

\section{Literatur}

1 Dummer R (beratend haben mitgewirkt: Andreesen R, Hermanek P, Höffken K, Moll R, Sauer H, Wollina U). Kutane Lymphome. AWMFLeitlinienregister Nr. 032/027 (Aktualisierung Oktober 2001); Dt. Krebsgesellschaft: Kurzgefasste Interdisziplinäre Leitlinien 2002, 3. Auflage 2002

2 Dummer R, Kempf W, Hess Schmidt M, Häffner A, Burg G. Therapy of cutaneous lymphoma-current practise and future developments. Onkologie 2003; 26:366-372

3 Howard MS, Smoller BR. Mycosis fungoides: classic disease and variant presentations. Semin Cutan Med Surg 2000; 19:91 - 99

4 Von Nida J, Randell P, Heenan P. Granulomatous mycosis fungoides with extensive chest wall involvement. Australas J Dermatol 2004; $45: 42-46$

5 Wollina U, Dummer R, Brockmeyer NH, Konrad H, Busch J-O, Kaatz M, Knopf B, Koch H-J, Hauschild A. Multicenter study of pegylated liposomal doxorubucin in patients with cutaneous T-cell lymphoma. Cancer 2003; $98: 993-1001$

6 Wollina U, Graefe T, Füller J. Granulomatous slack skin or granulomatous mycosis fungoides - a case report. Complete remission to percutaneous radiation and interferon alpha. J Cancer Res Clin Oncol 2002; $128: 50-54$ 
Mycosis fungoides im Plaque-Stadium (T1 NO M0 B0, Stadium Ia nach UICC 1993) - eine Indikation zur Strahlentherapie

\section{A. Laumanns, N. Christen, J. Schorcht , U. Wollina}

\section{Anamnese}

Seit Anfang des Jahres 2003 fielen der 76-jährigen Patientin juckende, plaqueartige Hautveränderungen an der linken Schläfe und am mittleren Rücken rechts auf.

\section{Hautbefund}

Plaques mit livid-roter Färbung, indolent, mit deutlicher epidermaler Atrophie (Abb.4a). Am Rücken Neigung zu Krustenbildung, jedoch keine Blutung, kein Nässen, kein Brennen.

\section{Laborparameter und bildgebende Diagnostik}

Kein Hinweis auf systemische Ausbreitung oder extrakutane Lymphome.

\section{Histologie}

Dichtes sowohl oberflächlich und tief perivaskulär als auch subepidermal bandförmig interstitiell (lichenoid) lokalisiertes Infiltrat. Infiltratepidermotropismus. Ausbildung typischer Pautrier'scher Mikroabszesse. Epidermale Infiltratzellen erscheinen größer als die dermalen und weisen Kernatypien auf.

\section{Therapie und Verlauf}

Der Rückenherd wurde im Rahmen der histologischen Abklärung exzidiert. Nach initialer topischer Steroidanwendung am Schläfenherd partielle Response. Im Dezember 2003 jedoch Progress an der linken Schläfe und Auftreten einer neuen Läsion am linken Nasenflügel. Daraufhin Röntgenbestrahlung unter Röntgentiefentherapiebedingungen mit $40 \mathrm{kV}$ dieser Region mit insgesamt 30,0 Gy in 15 Einzelfraktionen à 2,0 Gy/OD. Darunter Komplettremission (Abb. 4 b).

Bei einer erneuten ambulanten Vorstellung der Patientin Anfang März 2004 sahen wir eine reizlose Narbe am Rücken und eine infiltratfreie linke Gesichtshälfte mit geringem Erythem und leichter Epilation des angrenzenden Schläfenbereichs.

\section{Kommentar}

Die Mycosis fungoides gehört zu den niedrig-malignen primären T-Zell-Lymphomen und ist mit ca. 70\% die häufigste Entität. Standardtherapieoptionen der Mycosis fungoides im Plaque-Stadium sind lokale Steroidapplikation (Klasse III), UVB-Breitband/ UVB 311 nm, PUVA (auch in Kombination mit Interferon-alpha, Acitretin oder Bexaroten) und lokoregionale Orthovolt-Weichstrahlröntgentherapie [1,2]. Gerade bei therapieresistenten oder tumorösen Einzelherden zeigt die Weichstrahl-Röntgentherapie exzellente Ergebnisse bei geringen, lokalen Nebenwirkungen. Lange rezidivfreie Intervalle wurden beobachtet [2].

\section{Literatur}

1 Dummer R, Kempf W, Hess Schmidt M, Häffner A, Burg G. Therapy of cutaneous lymphoma-current practise and future developments. Onkologie 2003; 26:366-372

2 Micaily B, Miyamoto C, Kantor G, Lessin S, Rook A, Brady L, Goos, Vonderheid EC. Radiotherapy for unilesional mycosis fungoides. Int J Radiat Oncol Biol Phys 1998; 42:361-364
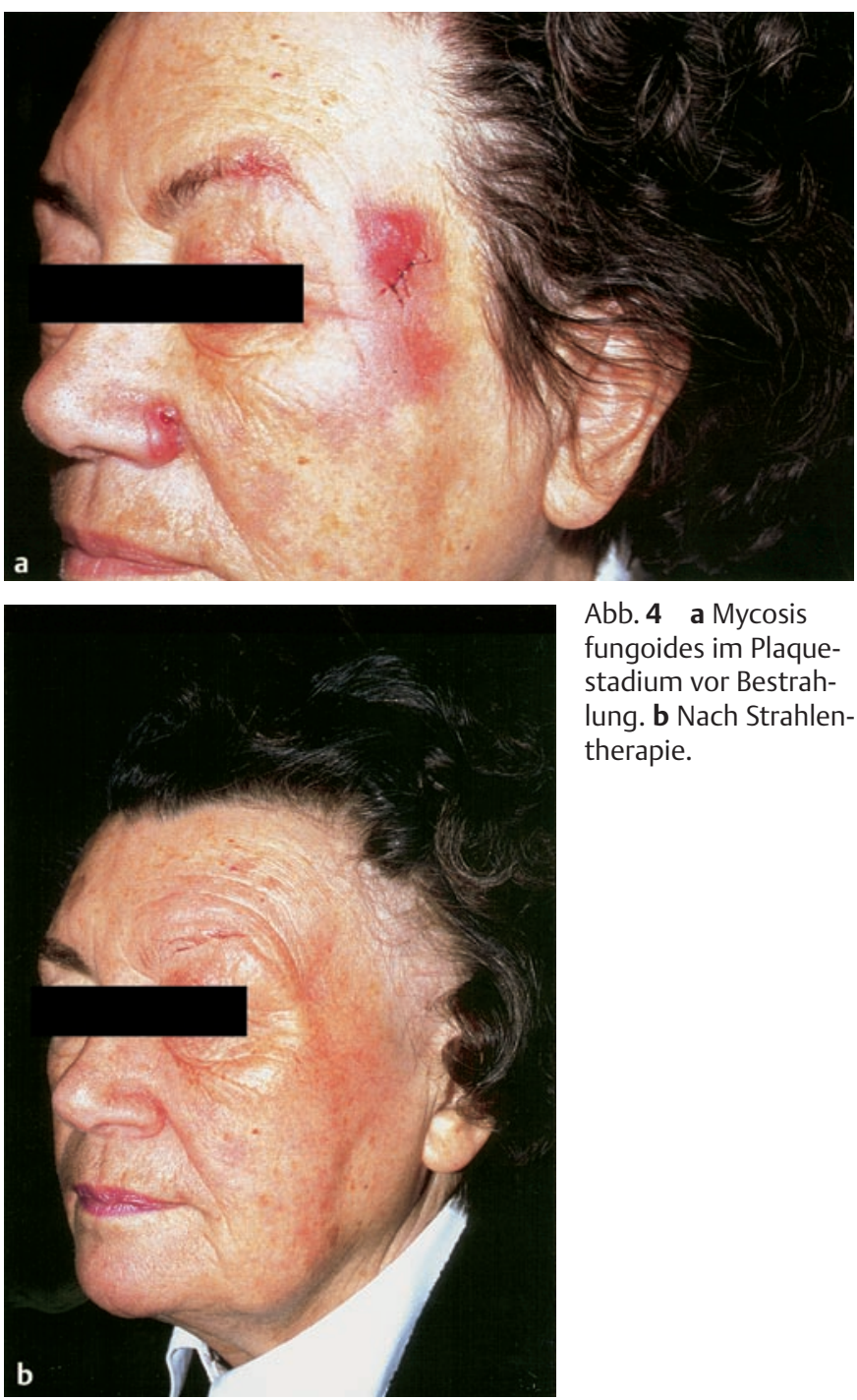

Abb. 4 a Mycosis fungoides im Plaquestadium vor Bestrahlung. b Nach Strahlentherapie.

Atypisches rezidivierendes Basalzellkarzinom der Wange vor Lebertransplantation - operative Strategien

\section{A. Laumanns, H. Konrad, E. Köstler}

\section{Anamnese}

Vorstellig wurde eine 65-jährige Patientin vor Lebertransplantation mit Rezidiv eines andernorts zweimalig voroperierten Basalzellkarzinoms der Wange. Medikamentöse Dauertherapie: Prednisolon 5 mg/die, Ciprobay, Spironolacton, Kalzium, Dekristol.

\section{Lokalbefund}

Ca. $3 \mathrm{~cm}$ im Durchmesser messende hypopigmentierte leicht atrophische, zentralulzerierte Plaque der linken Wange (Abb. 5a).

\section{Therapie und Verlauf}

Nach Abklärung der Diagnose und Ausdehnung durch Entnahme von fünf $2 \mathrm{~mm}$-Stanzbioptaten des Randes mit histologischer Sicherung des Basalzellkarzinoms, nachfolgende zweizeitige Exzision des Tumors mit $4 \times 3,5 \mathrm{~cm}$ großem Defekt. Nach histologischer Schnittrandkontrolle nochmalige Nachexzision zwischen 9 und 12 Uhr (Ergebnis: histologisch tumorfrei) und Defektdeckung durch ausgedehnte Wangenrotationsplastik in Lokalanäs- 

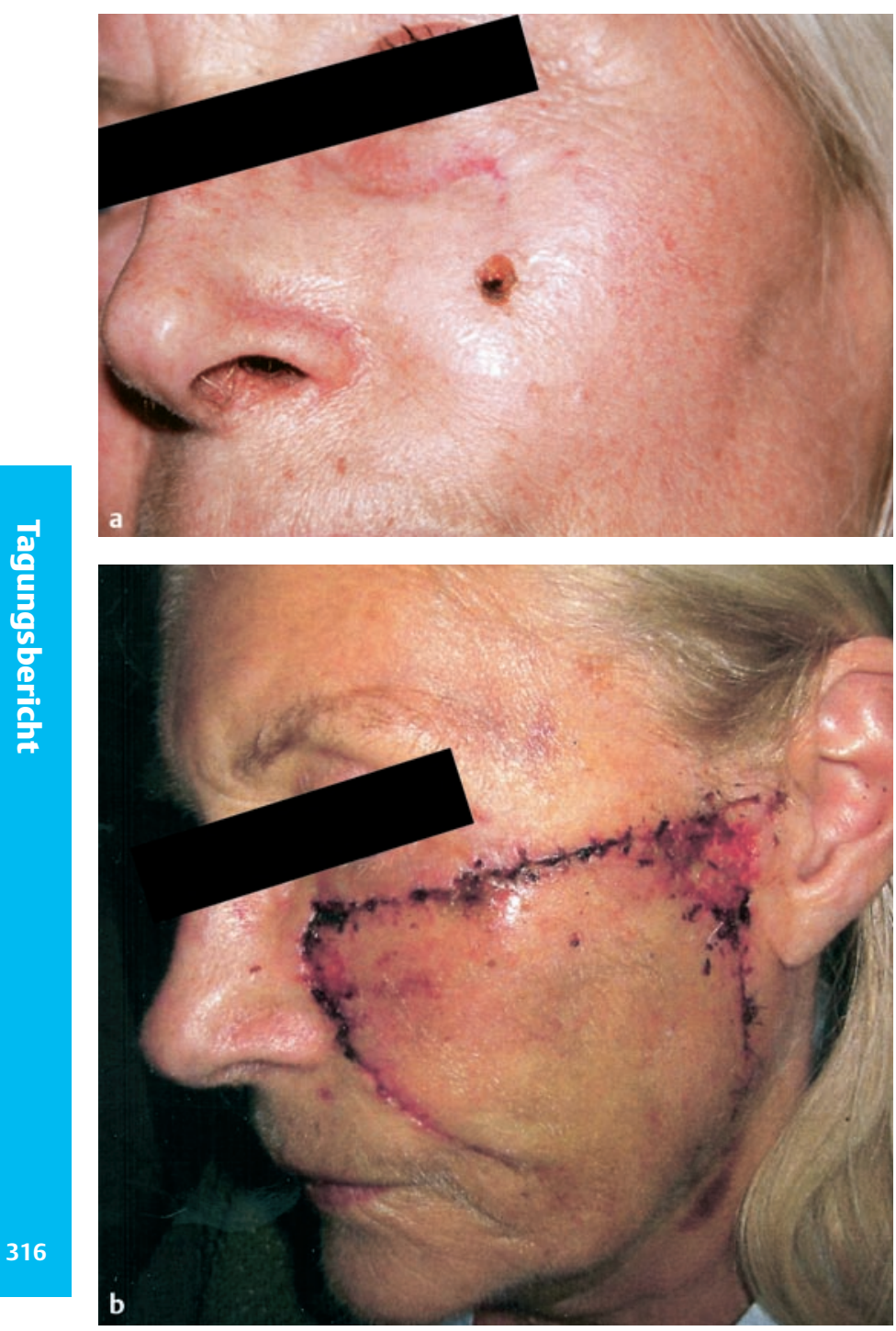

Abb 5 a Atypisches Basalzellkarzinom. b Postoperativer Befund.

thesie kombiniert mit kleinem nasolabialen Verschiebelappen und kleinem Vollhauttransplantat aus der Nasolabialfalte. Gute Einheilung bis auf einen kleinen oberflächlichen nekrotischen Anteil im kranialen Bereich des Verschiebelappens (Abb. 5b). Kontrollbefund nach einem Jahr ohne Anhalt für Tumorrezidiv mit gutem ästhetisch-funktionellem Ergebnis.

\section{Kommentar}

Das Basalzellkarzinom ist durch eine Entkopplung der Wachstumskontrolle über den TGF-beta-Rezeptor und intrazelluläre Smad-Proteine gekennzeichnet [3]. Hierdurch kann ein relativ autonomes Wachstum mit lokaler Infiltration und Destruktion stattfinden. Marker der späten epidermalen Differenzierung, wie die Expression des Keratins K2e, gehen in diesen Tumoren verloren [1].

Die Behandlung des Basalzellkarzinoms sollte deshalb idealer Weise eine vollständige Entfernung des Tumors, ein gutes kosmetisches Ergebnis mit Erhaltung der Funktion und Maßnahmen zur Prophylaxe (Nachkontrollen, Inspektionen) beinhalten [2, 4].
In der Wangenregion sind die technisch einfacheren freien Hauttransplantate aus ästhetischen Gründen weitgehend zu vermeiden. Insbesondere für Rezidive und schlecht abgrenzbare Tumoren und für solche mit sklerodermiformem und aggressiv infiltrativem Wachstum ist die mikroskopisch kontrollierte Chirurgie anzuwenden. Rezidivraten zwischen 1,7 und $6,5 \%$ werden erreicht. Patienten mit Dauerimmunsuppression - etwa nach Organtransplantation - entwickeln vermehrt Hautkrebse. Vorliegend konnte ein atypisches rezidivierendes Basalzellkarzinom unter Anwendung verschiedener plastischer Operationsverfahren funktionell und ästhetisch R0-resesziert werden. Weitere Tumorüberwachung ist jedoch notwendig.

\section{Literatur}

1 Bloor BK, Tidman N, Leigh IM, Odell E, Dogan B, Wollina U, Ghali L, Waseem A. Expression of Keratin K2e in cutaneous and oral lesions. Association with keratinocyte activation, proliferation, and keratinisation. Am J Pathol 2003; 162: $963-975$

2 Breuninger H, Garbe C. Basaliom. In: Korting HC, Callies R, Reusch M, Schlaeger M, Sterry W (Hrsg). Dermatologische Qualitätssicherung. Leitlinien und Empfehlungen. München: W Zuckschwerdt, 2003, $161-164$.

3 Lange D, Persson U, Wollina U, Ten Dijke P, Castelli E, Heldin C-H, Funa K. Expression of TGF-ß related Smad proteins in human epithelial skin tumors. Int J Oncol 1999; 14: 1049-1056

4 Sentürk GB, Hohenleutner S, Landthaler M et al. Mikrografische Chirurgie beim Basalzellkarzinom: 5-Jahres-Rezidivraten mit „LifeTable“-Analyse. Fortschr Operat Onkol Dermatol 2003; 19:31 - 34

\section{Paraneoplastische Dermatomyositis bei Adenokarzinom des} Kolons und Mikrosatelliten-Instabilität

\section{A. Koch, G. Kretschmer}

\section{Anamnese}

Im Mai 2003 traten erstmals im Gesicht des 38-jährigen Patienten Erytheme auf, die sich auf die Schulterregion ausweiteten. Subjektiv bestand eine gewisse Abgeschlagenheit und Mattigkeit mit muskulärer Schwäche im Schultergürtelbereich. In der Familie mehrfach Kolon- und Mamma-Karzinome.

\section{Hautbefund}

Es zeigte sich eine diffuse, teilweise livide Rötung und Schwellung des Gesichtes mit Heliotroperythem der Augenlider. Ebenfalls mitbetroffen waren Hals und oberer Thorax. Über den Fingerknöcheln sahen wir erythematöse leicht hyperkeratotische Papeln im Sinne des Gottron-Zeichens (Abb.6a). In Ergänzung des typischen Bildes fielen auch Teleangiektasien sowie Hämorrhagien am Nagelfalz auf.

\section{Labor}

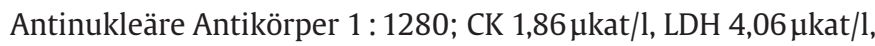
CEA $2,62 \mu \mathrm{g} / \mathrm{l}$.

\section{Elektromyogramm}

M. deltoideus: Erhöhte Polyphasierate, lebhafte Insertionsaktivität, niedrige Amplitude, fehlende Spontanaktivität.

\section{Histologie}

Hautbiopsie Gesicht: Epidermis abschnittsweise gering atrophisch, herdförmig mit diskreter Basalzelldegeneration. Schütte- 

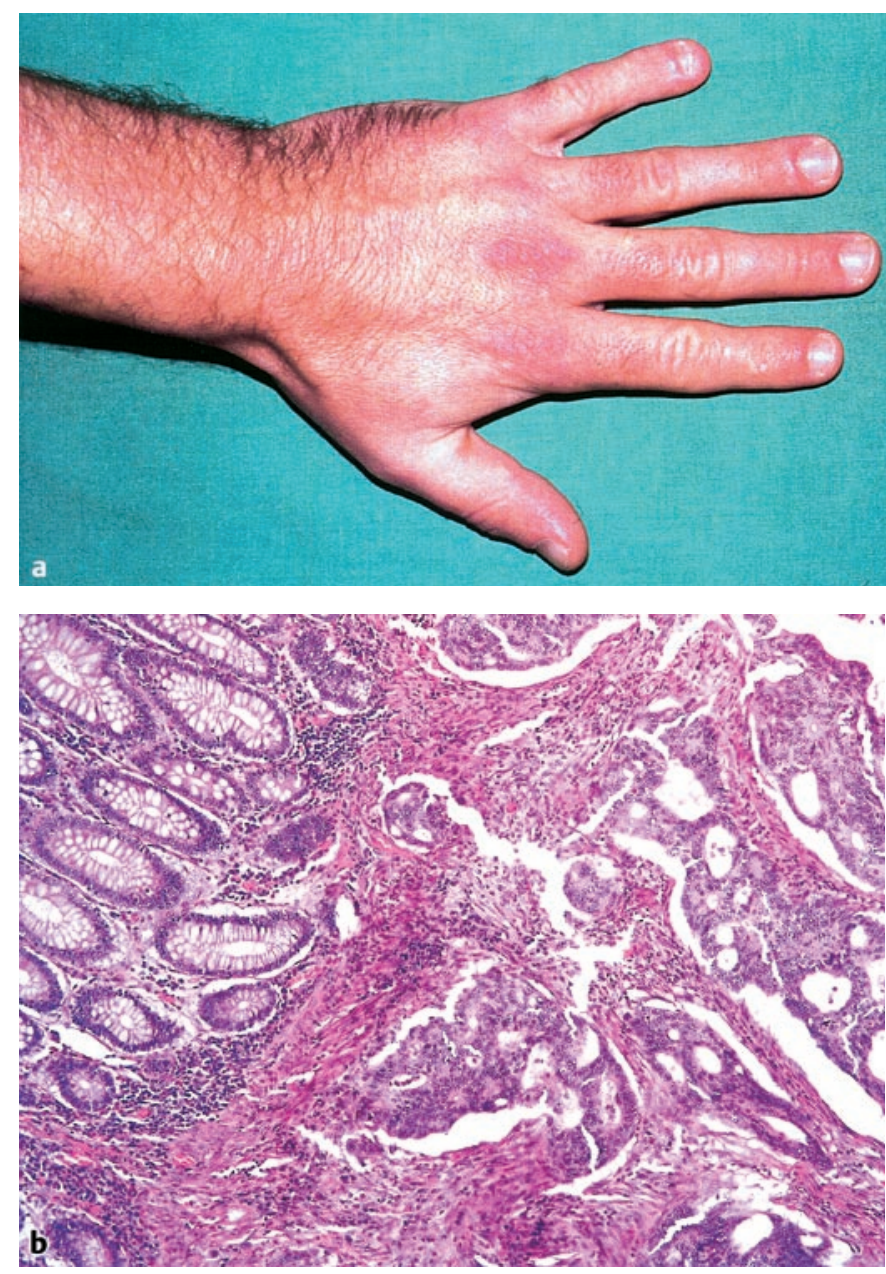

Abb. 6 a Gottron'sches Zeichen bei paraneoplastischer Dermatomyositis. b Adenokarzinom.

res, entzündliches, überwiegend lymphozytäres Infiltrat. Herdförmige Muzinablagerungen in der retikulären Dermis. Kein Lupusband.

Biopsie Caecum: Mäßig differenziertes tubulo-papilläres Adenokarzinom mit Lymph- und Hämangiosis carcinomatosa sowie Lebermetastase, G2, pT3 pN1 pM1 (Abb. 6b).

\section{Therapie}

Nach histologischer Sicherung des Caecumkarzinoms erfolgte die Verlegung in die Chirurgische Klinik zur Hemikolektomie. Intraoperativ fiel ein Leberrundherd auf, der ebenfalls entfernt wurde und sich als Metastase erwies. Dieser Rundherd war im Vorfeld sonografisch nicht gefunden worden. Im weiteren Verlauf ist eine Chemotherapie geplant.

\section{Kommentar}

Die „Dermatomyositis“ ist eine systemische Bindegewebserkrankung durch entzündliche und degenerative Veränderungen in den Muskeln mit charakteristischer Hautbeteiligung, die zu symmetrisch verteilter Muskelschwäche und mäßiger Muskelatrophie, vor allem der Gliedmaßengürtel, führt. Die Krankheit ist mit einer Häufigkeit von weniger als 1:100 000 relativ selten, wobei das Verhältnis Frauen zu Männer 2:1 beträgt. Zur Diagnose führen das charakteristische klinische Bild, erhöhte Muskel- enzyme im Serum, EMG-Veränderungen und die Muskelbiopsie (falls erforderlich).

Bei etwa einem Drittel der Patienten tritt eine Dermatomyositis als paraneoplastisches Syndrom auf, vor allem bei Ovarial- und Mammakarzinomen. Eine Tumorsuche ist bei Patienten älter als 35 Jahre notwendig [1]. Bei unserem Patienten war bereits die Häufung von Tumoren in der Familienanamnese auffällig. Es konnte eine Mikrosatelliteninstabilität nachgewiesen werden, was die erhöhte Tumorrate in der Familie erklären könnte. Mit Entfernung des Tumors im Caecum kam es zu einer deutlichen Besserung des Hautbildes.

\section{Literatur}

1 Willmer A, Lange D, Mentzel T, Wollina U. Paraneoplastische Derma-

\section{Lymphomatoide Papulose, Typ A}

\section{Gruner, I. Bach, J. Schönlebe}

\section{Anamnese}

Im Dezember 2003 bemerkte der 21-jährige Patient erstmalig vier diskret brennende Papeln am Thorax, die sich nach Ulzeration dann allmählich unter Hinterlassung einer Narbe zurückbildeten. Es sind weiterhin ein angeborener Protein-C-Mangel sowie ein resorptiv bedingter Eisenmangel infolge Magenschleimhautatrophie unklarer Genese bekannt.

\section{Hautbefund}

Ulzeronekrotische Herde mit einem durchschnittlichen Durchmesser von $1 \mathrm{~cm}$, daneben einzelne frische rötliche Papeln sowie narbig abgeheilte Herde disseminiert am gesamten Körper (Abb. 7a).

\section{Histologie}

Wechselnd dichtes teils perivaskulär, teils periadnexiell und interstitiell lokalisiertes Infiltrat, bestehend aus überwiegend reifen Lymphozyten, untermischt von monozytoiden Zellen, einzelnen Plasmazellen, wenigen neutrophilen Granulozyten und ganz vereinzelten Eosinophilen. Des Weiteren im Infiltrat herdbetont recht zahlreiche großleibige (blastäre) lymphoide Zellen mit atypischen Zellkernen (ca. 10-20\%). Diese Zellen lassen sich als T-Zellen identifizieren (CD3+) und exprimieren CD 30 (Abb. 7 b). Direkte Immunfluoreszenz: kein Nachweis spezifischer Immunreaktionen für IgA, IgG, IgM, Fibrinogen, C3, C4 und C1q.

\section{Therapie und Verlauf}

Äußerlich Glukokortikoide sowie Creme-PUVA, darunter bisher Teilremission.

\section{Kommentar}

Die lymphomatoide Papulose (LP) ist eine chronisch-rezidivierende Krankheit, deren überwiegend benigner klinischer Verlauf im Gegensatz zu der maligne erscheinenden Histologie steht. Sie wird als niedrig-malignes T-Zell-Lymphom eingeordnet. Bei etwa $10 \%$ der Patienten kommt es zu Übergängen in höher maligne kutane Lymphome, auch eine Assoziation zum Morbus Hodgkin ist beschrieben worden [1]. 

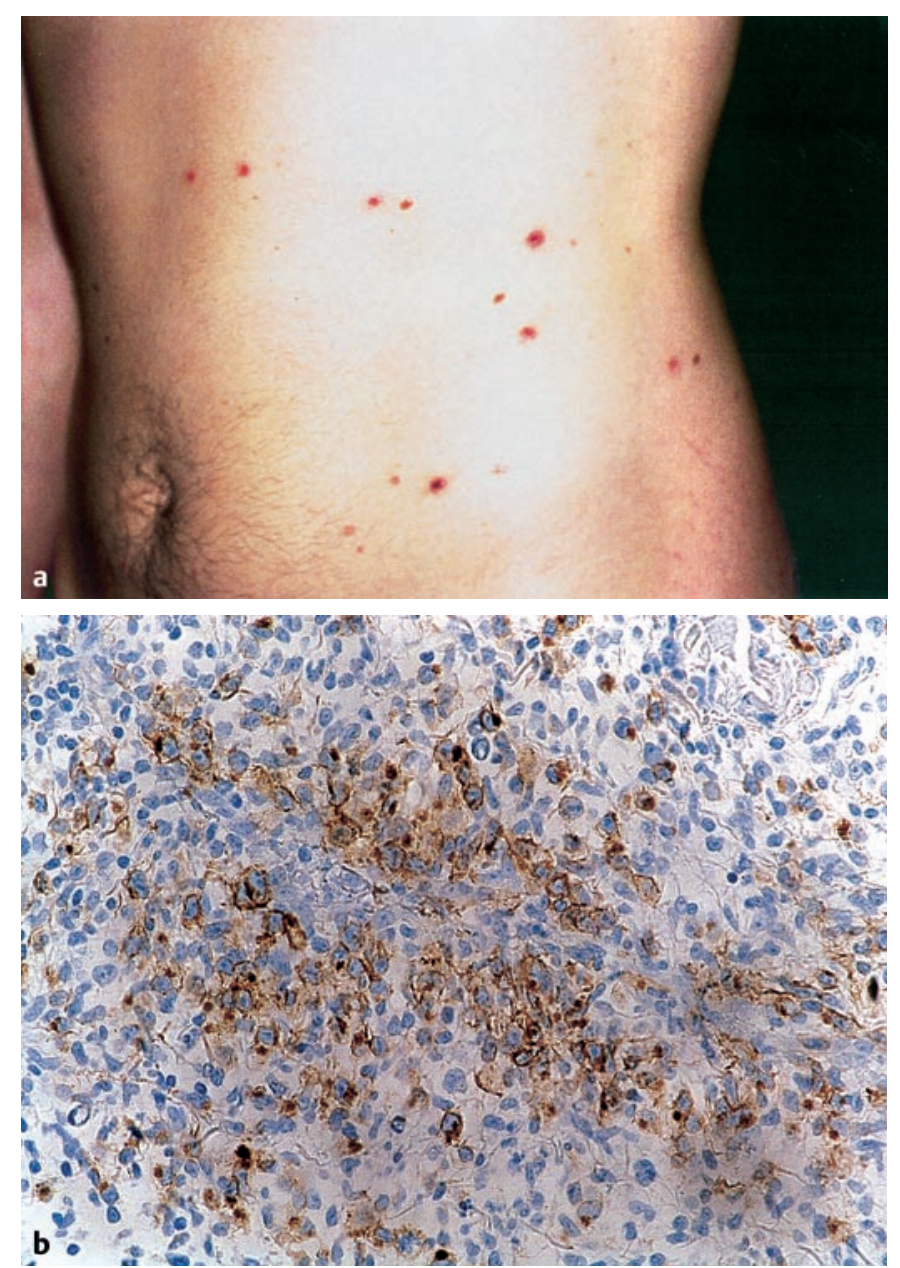

Abb. 7 a Lymphomatoide Papulose: Teils nekrotische Papeln, disseminiert einzeln stehend. b Lymphomatoide Papulose: CD30-positive Zellen - überwiegend in Sheets angeordnet.

Die Therapie der Wahl sind Photochemotherapie und/oder lokale Glukokortikoide. Therapierefraktäre Fälle können auch mit niedrig dosiertem Methotrexat behandelt werden. Extrakorporale Photopherese ist offenbar nicht so effektiv wie bei anderen kutanen Lymphomen [2]. Aufgrund des erhöhten Risikos, an einem malignen Lymphom zu erkranken, ist eine lebenslange engmaschige Kontrolle der Patienten ratsam.

\section{Literatur}

1 Orchard GE. Lymphomatoid papulosis: a low-grade T-cell lymphoma? Br J Biomed Sci 1996; $53: 162$ - 169

2 Wollina U. Lymphomatoid papulosis treated with extracorporeal photochemotherapy. Oncol Rep 1998; 5:57-59

\section{Still-Syndrom des Erwachsenen}

\section{Krönert}

\section{Anamnese}

Im Mai 2003 traten bei dem 24-jährigen Patienten akut grippeartige Beschwerden mit Halsschmerzen und Fieber auf. Vier Monate später bemerkte der Patient wenig juckende Hautveränderungen, die zunächst unregelmäßig, im weiteren Verlauf all- abendlich auftraten. In der Folge traten Nachtschweiß, Schüttelfrost, nächtliche Fieberattacken um $40^{\circ} \mathrm{C}$, Gelenk- und Muskelschmerzen hinzu.

Aufgrund dieser Symptome und der zusätzlichen Lymphadenopathie konsultierte der Patient seinen Hausarzt, der eine Klinikeinweisung in eine auswärtige Innere Klinik veranlasste. Nach umfangreichen diagnostischen Maßnahmen (Infektionsserologie, Röntgen, Sonographie, Echokardiographie inkl. TEE), die Lymphknoten-Schwellungen zervikal und eine Splenomegalie belegten, konnten letztlich keine Ursachen für die rezidivierenden Fieberschübe, die Arthralgien und das Exanthem eruiert werden. Die durchgeführte antibiotische Therapie erbrachte keine wesentliche Befundbesserung. Im Januar 2004 Aufnahme in die Hautklinik Dresden-Friedrichstadt.

\section{Hautbefund}

Abendliche hellrote zum Teil konfluierende Makulae und kaum erhabene Quaddeln an Stamm, Oberschenkelinnenseiten, Handrücken und Unterarmen (Abb. 8). Schleimhäute unauffällig. Lymphknotenschwellung zervikal.

\section{Histologie}

Im Bereich des oberflächlichen Gefäßplexus ein nur außerordentlich schütteres perivaskulär lokalisiertes lymphomonozytoides Infiltrat mit wenigen Mastzellen. Kein sonstiger nennenswerter pathologischer Befund. Direkte Immunfluoreszenzuntersuchung negativ.

\section{Laborbefunde}

Ferritin $712 \mu \mathrm{g} / \mathrm{l}$, Eisen $6,24 \mu \mathrm{mol} / \mathrm{l}$, CRP $35,7 \mathrm{mg} / \mathrm{l}$, LDH 4,32 $\mu \mathrm{kat} / \mathrm{l}$, Blutbild, Lymphozytendifferenzierung, ANA, RF, Cardiolipin-AK, Komplement, Immunfixation, IL-2-Rezeptor, Toxoplasmose-, Hepatitis- und Rubellaserologie unauffällig.

\section{Therapie und Verlauf}

Unter antiphlogistischer Therapie mit Diclofenac in Kombination mit Prednisolon in mittlerer Dosierung konnte ein rasches Ansprechen mit Rückgang der Gelenk-, Muskelbeschwerden und der Hautveränderungen verzeichnet werden. Der Patient blieb konstant fieberfrei. Eine Komplettremission wurde erzielt.

\section{Kommentar}

Febrile Polyarthralgien resp. -arthritiden sind Symptome unterschiedlicher Krankheitsbilder. Schließt man Autoimmunerkrankungen, wie die rheumatoide Arthritis und die klassischen Kollagenosen (vor allem den SLE) sowie Infektionserkrankungen aus, sollte frühzeitig auch der Morbus Still des Erwachsenen - im Angloamerikanischen „adult-onset Still's disease (AOSD)“ - in die Differentialdiagnose einbezogen werden. Die Erkrankung ist selten - im Gegensatz zur kindlichen Form - fällt aber durch eine typische Symptomatolgie auf. Obligat finden sich septische intermittierende Fieberschübe bei Polyarthritis. Diagnostisch wegweisend sind das typische flüchtige gegen Abend auftretende lachsfarbene makulöse oder makulo-urtikarielle Exanthem mit Prädilektion an Stamm und proximalen Extremitäten sowie eine prodromale Pharyngitis. Charakteristisch ist ferner eine exzessive Hyperferritinämie [2]. Die Hauterscheinungen per se lassen natürlich auch an eine Urtikaria sowie urtikarielle (Virus- oder Arzneimittel-)Exantheme und Schnitzler-Syndrom denken. We- 


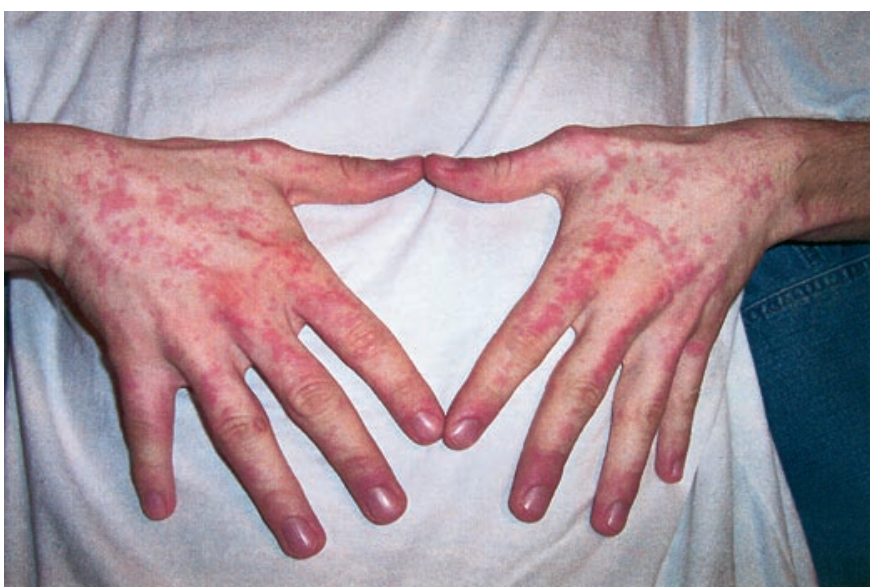

Abb. 8 Still-Syndrom des Erwachsenen, flüchtige urtikarielle Erytheme.

gen der Fieberschübe ist an ein Sweet-Syndrom, aufgrund der Arthritis an die interstitielle granulomatöse Dermatitis mit $\mathrm{Ar}$ thritis zu denken.

Vom japanischen Adult Still's Disease Research Committee [5] wurden Diagnosekriterien erarbeitet (Tab.1). Treffen 5 Kriterien, darunter mind. 2 Majorkriterien zu, kann die Diagnose AOSD mit hoher Wahrscheinlichkeit getroffen werden (ab 7 Kriterien nahezu 100\%) [3]. Früher wurde die Erkankung wahrscheinlich als Subsepsis allergica Wissler eingeordnet.

Die Ätiologie der seltenen AOSD ist unklar, Viruserkrankungen als auslösende Faktoren werden diskutiert. Der Altersgipfel der Erkrankten liegt zwischen dem 16. und 35. Lebensjahr. Die Prognose wird von der möglichen Nierenamyloidose geprägt.

Die Therapie erfolgt symptomatisch mit NSAR meist in Kombination mit Steroiden. Rezidivieren oder persistieren die Symptome z.B. nach Steroidreduktion, kommen neben Chloroquin oder Sulfasalazin auch Azathioprin, MTX, Cyclosporin oder Immun-

Tab. 1 Diagnosekriterien des Still-Syndroms des Erwachsenen

\begin{tabular}{|c|c|}
\hline \multicolumn{2}{|l|}{ Hauptkriterien } \\
\hline & Fieber $\geq 39^{\circ} \mathrm{C} \geq 1$ Woche $^{*}$ \\
\hline & Arthralgien $\geq 2$ Wochen (meist große Gelenke)* \\
\hline & Leukozytose mit $\geq 80 \%$ Neutrophilen \\
\hline & Abendliches makulöses lachsfarbenes Exanthem* \\
\hline \multicolumn{2}{|l|}{ Nebenkriterien } \\
\hline & Halsschmerzen* \\
\hline & Lymphadenopathie und/oder Splenomegalie* \\
\hline & Negative Rheumafaktoren, neg. ANA* \\
\hline & Erhöhte Transaminasen oder LDH* \\
\hline \multicolumn{2}{|l|}{ Ausschluss von } \\
\hline & $\begin{array}{l}\text { Malignomen (v. a. Lymphome), Infektionen, rheumatischen } \\
\text { Erkrankungen }\end{array}$ \\
\hline
\end{tabular}

${ }^{*}$ bei unserem Patienten zutreffend. globuline in Frage [3, 4]. Kürzlich wurde über therapeutische Erfolge mit dem TNF-alpha-Inhibitor Infliximab berichtet [1].

Der Verlauf ist sehr variabel, sowohl Spontanheilungen als auch chronisch rezidivierende Fälle wurden beschrieben. Komplizierend können Serositiden, Nierenamyloidose und Gelenkdestruktionen hinzutreten.

\section{Literatur}

1 Kraetsch HG, Antoni C, Kalden JR, Manger B. Successful treatment of a small cohort of patients with adult-onset Still's disease with infliximab: first experiences. Ann Rheum Dis 2001; 60:55-57

2 Menge F, Schacherer C, Wigand R, Brodt R, Caspary WF, Stein J. Morbus Still des Erwachsenen. Differentialdiagnose bei rekurrenter Pharyngitis, Fieber unklarer Genese und exzessiver Hyperferritinämie. Dtsch Med Wochenschr 1999; 124:1541

3 Omagari K, Matsunaga Y, Yamashita H, Nishiyama H, Hazama H, Oda H, Isomoto H, Mizuta Y, Murase K, Kohno S. Successful treatment with cyclosporin in adult-onset Still's disease manifesting as acute hepatitis with marked hyperferritinemia. Am J Med Sci 2003; 326: 148-151

4 Sasaki R, Shiba H, Kin M, Ohyama M, Yamana J, Yamana S. Adult-onset Still's disease (AOSD) treated with a combination of prednisolone, cyclosporin A and methotrexate. Ryumachi 2003; 43:649-653

5 Yamaguchi M, Ohta A, Tsunematsu T, Kasukawa R, Mizushima Y, Kashiwagi H, Kashiwazaki S, Tanimoto K, Matsumoto Y, Ota T, et al. Preliminary criteria for classification of adult Still's disease. J Rheumatol. 1992; $19: 424-430$

\section{Schnitzler-Syndrom}

\section{A. Gemmeke, S. Balzer}

\section{Anamnese}

Seit über 30 Jahren leidet die 48-jährige Patientin an chronischrezidivierenden, disseminierten, urtikariellen Hautveränderungen. Seit zwei Jahren bestehen Gelenkbeschwerden der großen Gelenke und rezidivierende Fieberschübe mit mäßigem Juckreiz. Seitdem klagt die Patientin über Schwäche und Kraftlosigkeit.

\section{Hautbefund}

Generalisiertes urtikarielles, kleinfleckiges, hellrotes Exanthem mit Aussparung des Gesichtes, der Palmae und Plantae (Abb.9). Lymphknoten axillär beidseits palpabel.

\section{Histologie}

Perivaskuläre lymphohistiozytäre Infiltration im oberen bis tiefen Korium, teils bis in die anhängende Subkutis reichend. Einzelne eosinophile Granulozyten, verschiedene Mastzellen. Daneben vaskulitische Veränderungen mit Leukozytoklasie von Kapillaren sowie perivaskulären neutrophilen Granulozyten und Kerntrümmern. Negativer Eisennachweis. In der direkten Immunfluoreszenz IgM und IgG an Kapillarwänden.

\section{Laborbefunde}

BSG 42 mm, Leukozyten 11,6 gpt/l, Thrombozyten 552 gpt/l, Neutrophile 81,7\%, Neutrophile absolut 9,50 gpt/1, Lymphozyten 13,3\%, Immunglobulin M 6,06 g/l (Normwert: 0,4-2,3 g/l), in Serum und Urin Nachweis von IgM-Paraprotein. 


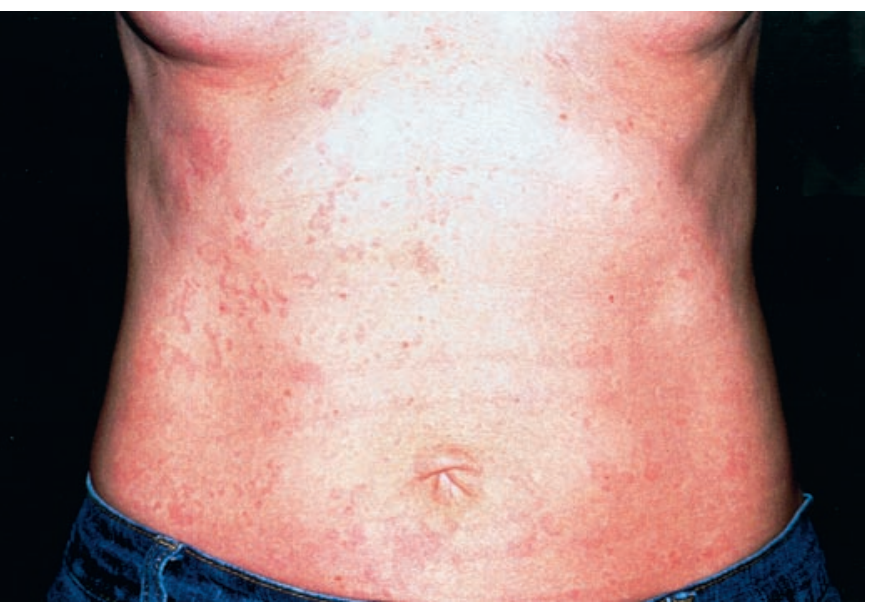

Abb. 9 Schnitzler-Syndrom: Urtikartielles Exanthem.

\section{Röntgen, CT, MRT und Knochenszintigraphie}

Kein Nachweis von Osteolysen oder Lymphomen. Geringgradige Mehrspeicherungen im Bereich beider Schultergelenke und im rechten Knie.

\section{Beckenkammzytologie und -histologie}

Kein Nachweis eines Plasmozytoms oder anderer Lymphome.

\section{Therapie}

Zur Zeit ohne.

\section{Kommentar}

Das Schnitzler-Syndrom ist eine seltene Erkrankung, die in ihrer vollen klinischen Symptomatik durch gemeinsames Auftreten von chronischer Urtikaria, monoklonaler IgM-Makroglobulinämie, intermittierenden Fieberschüben mit erhöhter Blutsenkung sowie hartnäckigen Knochen- und/oder Gelenkschmerzen charakterisiert ist. Häufig besteht ein jahrzehntelanger Verlauf bis zur Entwicklung des Vollbildes. Beide Geschlechter sind gleichmäßig betroffen. Initial ist meist eine chronische Urtikaria vorhanden, die meist kleinfleckig, rosafarben und unter Aussparung des Gesichtes, der Palmae und Plantae generalisiert auftritt und die nach Monaten bis Jahren von rheumatischen Beschwerden gefolgt ist. Typischerweise verläuft die Urtikaria ohne oder mit mäßigem Juckreiz. Der Allgemeinzustand der Patienten erscheint auffällig gut, lediglich während der Fieberattacken besteht eine Beeinträchtigung. Häufig werden indolente Lymphknotenvergrößerungen gefunden. Histologisch findet sich eine leukozytoklastische Vaskulitis [1,2]. Differenzialdiagnostisch müssen ein systemischer Lupus erythematodes, ein Morbus Waldenström, eine Kryoglobulinämie und ein neoplastisches Geschehen ausgeschlossen werden.

Äußerst schwierig gestaltet sich die Therapie. Antihistaminika erweisen sich meist als wirkungslos. Die Behandlung mit DADPS, Interferon-alpha, hochdosierten Immunglobulinen oder Cyclosporin wird in der Literatur kontrovers diskutiert. Kortikosteroide in hoher Dosierung bzw. in Kombination mit Kolchizin zeigten vereinzelt eine positive Beeinflussung der Urtikaria. Ibuprofen kann sich v. a. auf die Knochenschmerzen wie auch auf die Hautveränderungen und die Fieberschübe günstig auswirken $[3,4]$.
Die Prognose des chronisch verlaufenden Schnitzler-Syndroms ist gut. Langzeitbeobachtungen haben jedoch ergeben, dass sich nach vielen Jahren gelegentlich auch ein malignes Lymphom entwickeln kann. Aus diesem Grund sind langfristige Verlaufskontrollen bei allen betroffenen Patienten ratsam [1,2].

\section{Literatur}

1 Akimoto R, Yoshida M, Matsuda R, Miyasaka K, Itoh M. Schnitzler's syndrome with IgG kappa gammopathy. J Dermatol 2002; 29:735-738

2 Lautenschlager S, Itin PH. Das Schnitzler-Syndrom. Hautarzt 1993; 44:781-784

3 Pascual-López M, Hernández-Núnez A, Sánchez-Pérez J, FernándezHerrera J, García-Díez A. Schnitzler's syndrome with monoclonal IgG k gammopathy: good response to cyclosporin. J Eur Acad Dermatol Venereol 2002; 16:267-270

4 Schartz NE, Buder S, Sperl H, Audring H, Paus R, Tebbe B, Kruger K, Sterry W. Report of a case of Schnitzler's syndrome treated successfully with interferon alpha 2b. Dermatology 2002; $205: 54-56$

\section{Scleroedema adulltorum Buschke}

\section{A. Gemmeke, M. Gebhardt}

\section{Anamnese}

Seit 1996 leidet die 62-jährige Patientin an einer chronisch-persistierenden Schwellung des Nackens und der proximalen Oberarme. Des Weiteren klagt die Patientin über Bewegungseinschränkung im Schulterbereich. In der Vergangenheit ist die Patientin bereits hochdosiert mit Penizillin i. v. über 10 Tage, Prostavasin i. v. über drei Wochen und über drei Jahre mit der extrakorporalen Photophorese behandelt worden, die jeweils nur eine kurzfristige Befundbesserung ergaben.

Seit 27 Jahren ist ein insulinpflichtiger Diabetes mellitus Typ II mit Polyneuropathie und Retinopathie bekannt. Auch der Vater und vier Geschwister hätten an Diabetes mellitus Typ II gelitten. In augenärztlicher Betreuung ist die Patientin bei beidseitigem Glaucoma chronicum. Des Weiteren ist die Patientin wegen einer arteriellen Hypertonie in internistischer Behandlung. 1998 und 2003 erfolgte die Spaltung der Ligg. carpi transversa bei beidseitigem Karpaltunnelsyndrom.

\section{Befund}

Beginnender Morbus Dupuytren beidseits. Die Beweglichkeit in beiden Schultergelenken ist bei einer maximalen Elevation von $160{ }^{\circ}$ beidseits eingeschränkt. Der sonstige Gelenkstatus ist unauffällig.

Im Nacken und an den proximalen Oberarmen zeigt sich eine flächenhafte, derbe, ödematöse Schwellung der farblich unveränderten bis leicht lividen Haut. Hautfarbene lichenoide Papeln fanden sich nur andeutungsweise (Abb. 10a). Allerdings war die Patientin auch mehrfach vorbehandelt (s. oben). Die betroffenen Hautareale sind nicht eindrückbar und nicht in Falten abzuheben. Die Lateralseiten der Oberschenkel sind diskret verhärtet.

\section{Histologie}

Kollagenfasern der insgesamt beträchtlich verbreiterten Dermis verdickt und homogenisiert, durch Zwischenräume im Sinne der Fenestration voneinander getrennt. Gehalt an Fibroblasten 

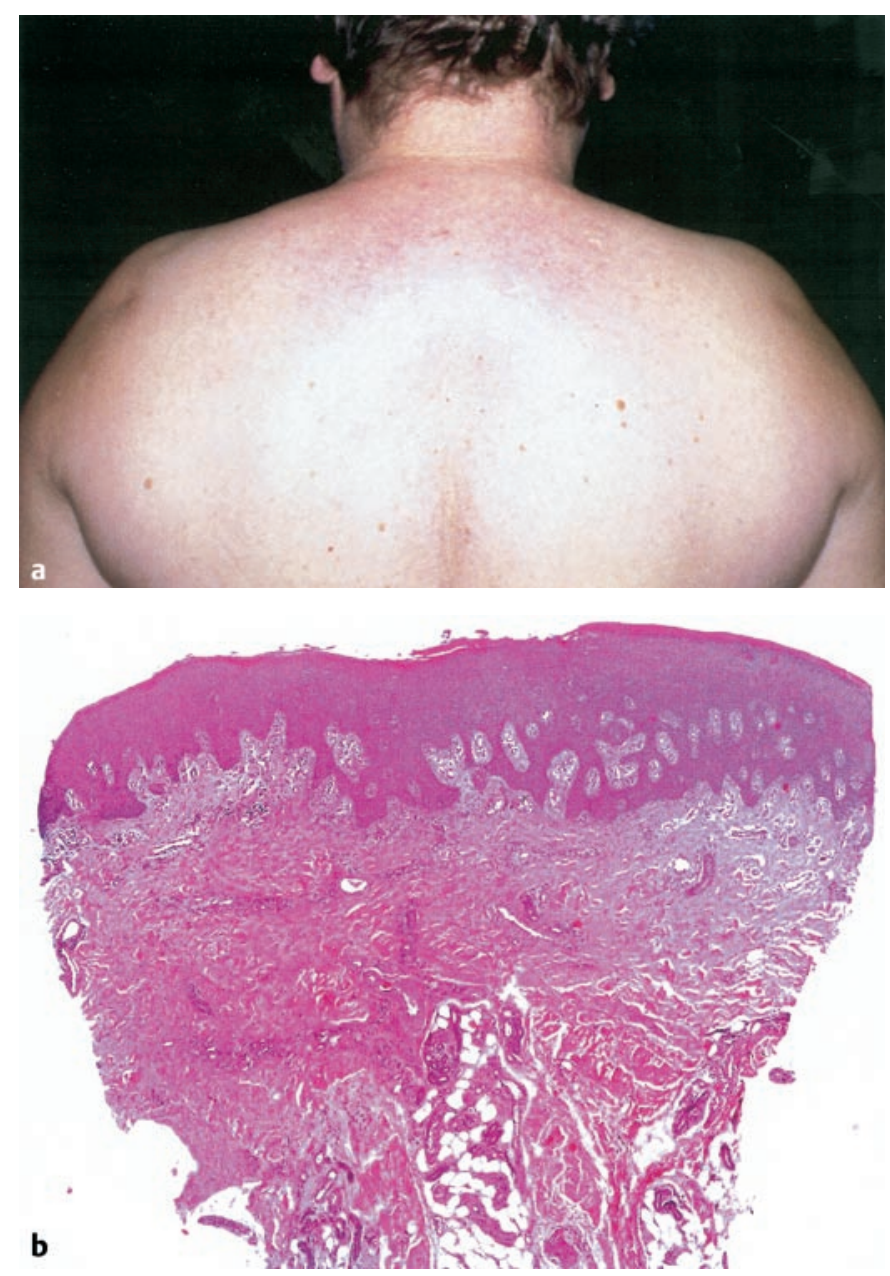

Abb. 10 a Scleroedema adultorum mit sklerodermiform verdickter Haut der Schulterregion. b Scleroedema adultorum. Histologie-Übersicht (HE).

vermindert. Ebenso Verminderung des Gehaltes an elastischen Fasern. Ekkrine Schweißdrüsen im mittleren Korium, eingemauert in kollagenes Bindegewebe, das offensichtlich das subkutane Fettgewebe verdrängt hat (Abb. 10b). In der mittleren und tiefen Dermis herdförmig positiver Nachweis interstitieller Muzinablagerungen zwischen den Kollagenfasern.

\section{Laborbefunde}

HbA1c 7,0\%, Glucose i. B. 6,02 mmol/l, Creatinin i. S. 96,2 umol/l, LDH 4,33 $\mu \mathrm{kat} / \mathrm{l}$, Parathormon 61,1 pg/ml. Durch den Dexamethason-Hemmtest konnte ein Hyperkortisolismus ausgeschlossen werden. Kein Nachweis von Paraprotein in Serum und Urin.

\section{Sonstige Befunde}

EKG und bildgebende Diagnostik unauffällig.

\section{Therapie}

Unter Photochemotherapie mit Bade-PUVA sowie intensiver physiotherapeutischer Behandlung mit Lymphdrainagen und Pelosepackungen war eine Besserung der Beweglichkeit und eine Abnahme der Hautsklerose zu verzeichnen.

\section{Kommentar}

Das Scleroedema adultorum Buschke ist eine seltene chronischprogrediente Bindegewebserkrankung unbekannter Ätiologie. Charakteristisch ist die flächenhafte, brettharte, ödematöse Schwellung der oberen Rumpfhaut mit Übergreifen auf die obere Extremität. Die Erkrankung kann in jedem Lebensalter, vor allem beim weiblichen Geschlecht auftreten. Der Diabetes mellitus ist offenbar ein prädisponierender Faktor, denn er wird bei 30-50\% aller betroffenen Kranken gesehen [4]. Eine Mitbeteiligung innerer Organe z. B. des Herzmuskel mit EKG-Veränderungen, der Augen mit Sicca-Symptomatik, des Ösophagus mit Schluckstörungen, ein begleitender Hyperparathyreoidismus oder eine Hepatomegalie sind selten. Eine Paraproteinämie muss ausgeschlossen werden, da hierbei progressive Verläufe Richtung Plasmozytom beschrieben sind.

Histologisch fallen ödematös verquollene Kollagenfasern im Korium und in der Subkutis mit Ansammlung von Mukopolysacchariden auf. Differenzialdiagnostisch sind die systemische Sklerodermie, das Skleromyxödem, das Stiff-Skin-Syndrom, andere Pseudosklerodermien und ein Morbus Cushing auszuschließen.

Der Krankheitsverlauf ist insgesamt meistens milde. Die Hautveränderungen können jedoch über Jahre persistieren. Eine Standardtherapie ist nicht bekannt. In Einzelfällen führten PUVA oder extrakorporale Photophorese zu einer deutlichen Besserung des Hautfundes [1, 2]. Hochdosierte Gaben von Penizillin oder von Kortikosteroiden können versucht werden. Auch die intermittierende Ciclosporin-A-Therapie oder eine Behandlung mit niedrigdosiertem Methotrexat wurden beschrieben [3, 5]. Physikalische Therapie mit Lymphdrainagen; Wärmeanwendung und Massagen sind die Basis, um eine weitergehende Einschränkung der Beweglichkeit zu verhindern.

\section{Literatur}

1 Grundmann-Kollmann M, Ochsendorf F, Zollner TM, Spieth K, Kaufmann R, Podda M. Cream PUVA therapy for scleredema adultorum. $\mathrm{Br}$ J Dermatol 2000; $142: 1058-1059$

2 Hager CM, Sobhi HA, Hunzelmann N, Wickenhauser C, Scharenberg R, Krieg T, Scharffetter-Kochanek K. Bath-PUVA therapy in three patients with sleredema adultorum. J Am Acad Dermatol 1998; 38:240-242

3 Krasagakis K, Hettmannsperger U, Trautmann C, Tebbe B, Garbe C. Persistent sleredema of Buschke in a diabetic: improvement with highdose penicillin. Br J Dermatol 1996; 134:593-606

4 Rho YW, Suhr KB, Lee JH, Park JK. A clinical observation of scleredema adultorum and its relationship to diabetes. J Dermatol 1998; $25: 103-107$

5 Seygermm B, van den Hoogen FHJ, de Mare S, van Haelst U, de Jong EMGJ. A patient with a severe scleroedema diabeticorum, partially responding to low-dose methotrexate. Dermatology 1999; 198: $177-179$

6 Ulmer A, Schaumburg-Lever G, Bauer J, Kötter I, Fierlbeck G. Scleroedema adultorum Buschke. Hautarzt 1998; 49:48-54 